\title{
The Effects of Selective Aerobic Exercise on Cardiovascular Fitness on Debre Markos Preparatory School Sedentary Female Students
}

\author{
Fenta Bitew \\ Department of Sport Science, Debre Markos University, P.o.box 269 Debre Markos ,Ethiopia
}

\begin{abstract}
The study was conducted to investigate the effect of aerobic exercise on improving cardiovascular fitness of Debre markos preparatory school at Debre Markos town.15 sedentary preparatory school female students were selected as the study subjects and their age range were from 16-18 years. All selected participants were participated in moderate intensity aerobic exercise for 8 consecutive weeks ( 3 days per week), 60 minutes duration per day.12 minutes run test and step test were used and pre, during and after training test were conducted on cardiovascular fitness variables. The data collected from the study was analyzed quantitatively using descriptive statistics of central tendency of mean and standard deviation and determined the difference between initial and final mean for participants. According to the analyzed data in 12 minutes run test 826.86 meter mean difference was recorded and in step test 28.13 minute per bet mean difference was recorded and also in 12 minutes run test $73.33 \%$ of participants located under good performance zone and $26.66 \%$ of participants located under marginal zone after training. Based on the findings, it can be concluded that moderate aerobic exercise has positive effect on cardiovascular fitness of sedentary female students.
\end{abstract}

Keywords: Aerobic exercise, improving cardiovascular fitness, sedentary females

DOI: $10.7176 / \mathrm{JHMN} / 79-03$

Publication date:August $31^{\text {st }} 2020$

\section{INTRODUCTION}

\subsection{Background of the Study}

Aerobic exercise is an activity that strengthens the lungs and heart and it improves your body's ability to use oxygen. It can improve your heart rate and blood pressure as well as your breathing (Lippincott Williams and wilikins,2013)

Cardiovascular fitness is important for all human beings irrespective of their age. According to Reddy, 2012 a given work may not be carried out if the required physical strengthen is not available. Fitness is the first and foremost things to enjoy the life fully. Regular physical activity; fitness and exercise are critically important for health and wellbeing of people of all. Whether they have participate vigorous exercise or some type of moderate health enhancing physical activity. Even for every old adults, mobility and functioning can be improved through physical activity (butler et.al.,1998)

Aerobic activity should be an integral part of every exercise prescription for an apparently health individual, but how much aerobic in relation to resistance training depends in large part on each client's current condition and his or her fitness goals. Whether working with someone who was previously sedentary or a world class athlete's fitness related goals should be precise possible and future objectives. Measurement to make to insure the clients is progressing towards those goals whether they are fat reduction, general fitness or competitive athletes (Ekekakis, Panteleimon 2004).

Appropriate regular daily physical activity is a major component in preventing chronic disease, along with a healthy date and not smoking for individuals. Daily physical activity is a powerful means of preventing chronic disease. A reliable experience and scientific evidence show that regular physical activity provides people both male and female of any conditions including disabilities with a wide range of physical, social and mental health benefits WHO (2003).

Aerobic exercise is some time known as cardio exercise that requires pumping of oxygenated blood by the heart to deliver oxygen to working muscle and stimulates the heart rate and breathing rate to increase in a way that can be sustained for the exercise session. There are different of aerobic exercise such as cardio machine, spinning, running, swimming, walking, jogging and dancing are aerobic exercise that can help to prevent the chance of developing some cancers, diabetes, depression and cardio vascular disease (Brick.L.1996)

Regular aerobic exercise will produce beneficial effects for many age groups providing the exercise is specific and appropriate to the level of fitness of the individual. Progressive exercise correctly performed will increase the level of fitness and improve health. It will al so create a sense of wellbeing produce greater energy and reduce the risk of developing many disease. Exercise makes demands on the body system over and above normal every day activities and as the result the system adopt anatomically and physiologically (Rosser, 2001).

Depending on the above scholars and reviews idea the main brain storming and interest of the researcher to 
conduct this research is investigated the effect of selective aerobic exercise on cardio vascular fitness on Debre Markos preparatory school sedentary female students.

\subsection{Statement of the problem}

Aerobic exercise is fundamentally important to conduct screening for health concerns especially for cardio vascular fitness, live reducing blood pressure, improving circulation efficiency, reducing the risk of diabetes and facilitating the flow of air in and out of lungs. Many research studies say physical exercises are important for the development of all physical fitness. But no research was done in Debre Markos preparatory school on female cardio related physical fitness problems.

Now a days in our country Ethiopia, because of sedentary life style most people are attacked by chronic disease such as diabetes, hypertension, coronary heart disease, asthma and some other upcoming disease. According to many research studies findings, physical inactivity is one of the cause for the development of chronic disease and poor health fitness. Similarly, in Debre Markos Preparatory school students are living sedentary life style due to poor culture of having regular physical exercise.

Therefore, the research tried to investigate the effect of selective aerobic exercise on cardio vascular fitness on Debre Markos preparatory school sedentary female students on those who continually conduct physical exercise by preparing fitness training plan in month and the result was examined through experimentation.

\subsection{Basic research questions}

The following research questions were raised to achieve the objective of the research.

1. Does aerobic exercise affect cardiovascular endurance?

2. What is the relationship between aerobic exercise and cardiovascular endurance?

3. How aerobic exercises affect cardiovascular endurance?

\subsection{Objective of the study}

\subsubsection{General Objective}

The overall aim of the research is to investigating the effect of aerobic exercise on cardiovascular endurance on Debre Markos Preparatory school sedentary female students.

\subsubsection{Specific objectives}

The following specific objectives were addressed in this research

1. To test the effect of aerobic exercise on cardiovascular fitness on Debre Markos preparatory school sedentary Female students.

2. To evaluate the relationship between selective aerobic exercise and cardiovascular fitness.

3. To find out how selective aerobic exercise affects cardiovascular fitness.

\subsection{Significance of the study}

The research has the following significances:

1. The primary significant of the research was the researcher confidently knows the effect of aerobic exercise on cardiovascular fitness.

2. Sedentary female students in Debre Markos Preparatory school to understand the effect of aerobic exercise on cardiovascular fitness.

3. A base line for further studies on aerobic exercise on cardiovascular fitness.

\section{Methods and Materials}

\subsection{Study area and period}

The study has been conducted in Debre Markos Town, which is located at North West of the capital city Addis Ababa with $300 \mathrm{~km}$ distance and $265 \mathrm{~km}$ south east of Bahir Dar; the capital of Amhara national regional stat. the town has $1380 \mathrm{ml}$ average annual rainfall and minimum and maximum temperature of $15^{\circ} \mathrm{C}$ and $22^{\circ} \mathrm{C}$ respectively. The study period was took place in 2011E.C/2019GC.

\subsection{Study Design}

Pre-test and post-test experimental research design was used. To arrive at the final conclusion and to examine whether selective aerobic exercise have positive effects on cardiovascular fitness or not, pre exercise during exercise and post exercise experimental tests were taken by the respective employed physical quality measurements. Data was collected from primary source. 
Table1: The study design layout

\begin{tabular}{|l|l|l|}
\hline No & \multicolumn{1}{|c|}{ Treatment } & \multicolumn{1}{c|}{ Aerobic Exercise program } \\
\hline 1 & Frequency & 3 days per week \\
\hline 2 & Total duration & 8 weeks \\
\hline 3 & Duration/session & $40-60$ minutes \\
\hline 4 & Intensity & Moderate (55-69 HR max) \\
\hline 5 & Exercise days & Thursday, Sunday and Tuesday \\
\hline 6 & Time of training & Morning \\
\hline
\end{tabular}

\subsection{Population of the study}

The targeted populations of the study were 45 female students in Debre Markos preparatory school. Hence, the study was concerned with the examination of effects of aerobic exercise on cardiovascular endurance in sedentary female students.

\subsection{Sample size and Sampling techniques}

Among the targeted population of 45 students total number of females who willingly registered to participate in this study was 15.Based on medical history, questionnaires as well as inclusion and exclusion criteria 30 participants were excluded and 15 sedentary female students were fulfill inclusion criteria. Inclusion and exclusion criteria in a sense those participants who were healthy according to medical history questionnaire and who were included the age of 16-18 years where included in this study because majority of students were found in this age . However, those who did not qualify were excluded. Therefore, 15 sedentary female students in Debre Markos preparatory school were selected as subject unit. Purposive sampling technique was used.

\subsection{Source of data and data collection instrument}

Primary source of experimentation on result was a source of data. The collected through cardiovascular fitness test especially 12 minutes run test and step test. All the participants were instructed the first two days to run 12 minutes without training and record the result of each participant, and also the second two days the participants were instructed step up and down on 12 inch bench for three minutes at a rate of 24 steps per minute and recorded the second of the participants.

After measuring cardiovascular fitness before training by using 12 minutes run test and step test, start a training for 8 consecutive weeks by preparing training plan and measured cardiovascular endurance during training for 4 weeks of aerobic exercise using 12 minutes run test and step test and recorded the score of the participants. After giving 2 month training, measured the cardiovascular fitness by 12 minutes run test and step test and recorded the source of the participants. Finally, the result of the cardiovascular endurance before training and after training was compared.

\subsection{Data Analysis}

Descriptive statistics of mean and standard deviation were used and quantitative data analysis techniques were used for the research. The collected data were analyzed, interpreted and tabulated into meaning full idea using tables with appropriate standards, and percentage.

\subsection{Ethical consideration}

Initially letter of permission was taken from Debre Markos University, Department of Sport Science and submitted to Debre Markos preparatory school. Participants on the study were willing and the researcher was explained the purpose of the study for every participant and obtain information consent before beginning of the test. Moreover, culture and traditional value of all the population were taken to an account.

\subsection{Testing Standards of 12-minute run}

\begin{tabular}{|l|l|l|l|l|}
\hline \multicolumn{5}{|l|}{} \\
\hline Classification & \multicolumn{1}{|c|}{$\mathbf{1 7 - 2 6}$} & $\mathbf{2 7 - 3 9}$ & $\mathbf{4 0 - 4 9}$ & $\mathbf{5 0 +}$ \\
\hline High performance zone & $2320+$ & $2160+$ & $2000+$ & $1840+$ \\
\hline Good fitness zone & $2000-2319$ & $1920-2159$ & $1840-1999$ & $1680-1839$ \\
\hline Marginal zone & $1840-1999$ & $1680-1919$ & $1600-1839$ & $1520-1679$ \\
\hline Low zone & $<1840$ & $<1680$ & $<1600$ & $<1520$ \\
\hline
\end{tabular}

\subsubsection{Testing Procedure of 12 minute run test}

- Locate an area where a specific distance is already marked, or school truck

- Use stopwatch or wristwatch to accurately time a 12-minute period. 
- $\quad$ For best result warm up prior to test, \& then run at a steady pace for the entire 12 minutes.

- Determine the distance that students can run in 12 minutes.

- Depending up on your age, locate your score in rating chart.

\subsection{Testing Standard of step test training in 60 second heart rate}

\begin{tabular}{|l|l|}
\hline \multicolumn{1}{|c|}{ Classification } & \multicolumn{1}{c|}{ 60 second heart rate } \\
\hline High -performance zone & 84 or less \\
\hline Good fitness zone & $85-95$ \\
\hline Marginal zone & $96-119$ \\
\hline Low zone & 120 and above \\
\hline \multicolumn{1}{|c|}{ Adopted from Concept of physical fitness active life style for wellness 10th edition, 2000 }
\end{tabular}

\subsubsection{Procedure of test 60 second heart rate}

- Warm up prior to exercise

- Step up and down on 12 inch bench for three minutes at a rate of 24 steps per minute. One step consists of four beats; that is "up with the lift foot up with the right foot, down with the lift foot, down with the right foot".

- Immediately after the exercise, sit down on the bench and relax. Don't talk.

- Locate your pulse or have another person locate it for you.

- 5 seconds the exercise ends, begin continuing your pulse. Count the pulse for 60 second.

- Your score is your 60 second heart rate.

\section{Data analysis and Interpretation}

To achieve the purpose of the study 15 female students from Debre Markos Preparatory school sedentary female students were selected as a subjects and their age was 16-18 years. Selective aerobic exercise was given for 8 consecutive weeks. The variables selected for this study were cardiovascular fitness. Pre, during and post-test were conducted for all 15 subjects on cardio vascular fitness and the score were recorded and then the collected data was analyzed quantitatively by descriptive statics of central tendency. The results for cardiovascular fitness are discussed below.

Table 2: Results of 15 subjects' in step test standards before, during and after training

\begin{tabular}{|l|l|l|l|l|l|l|}
\hline $\begin{array}{l}\text { Standard } \\
\text { classification }\end{array}$ & $\begin{array}{l}\text { Number of subject } \\
\text { before training }\end{array}$ & $\mathbf{\%}$ & $\begin{array}{l}\text { Number of subjects } \\
\text { during training }\end{array}$ & $\mathbf{\%}$ & $\begin{array}{l}\text { Number of subjects } \\
\text { after training }\end{array}$ & $\%$ \\
\hline $\begin{array}{l}\text { High performance } \\
\text { zone }\end{array}$ & - & - & - & - & - & - \\
\hline Good fitness zone & - & - & - & - & 11 & 73.33 \\
\hline Marginal zone & - & - & - & - & 4 & 26.66 \\
\hline Lower zone & $\mathbf{1 5}$ & $\mathbf{1 0 0}$ & $\mathbf{1 5}$ & $\mathbf{1 0 0}$ & $\mathbf{1 5}$ & $\mathbf{1 0 0}$ \\
\hline
\end{tabular}

According to table 4, there was no subject who has high performance, good fitness and marginal zone level before and during training. All participants were located in low zone but after training $73.33 \%$ of the participants located under good fitness zone and $26.66 \%$ of participants located under marginal zone. This result indicated that effective change was observed on participant's cardiovascular level when they are engaged in selective aerobic exercise.

Table 3: Mean value of 12 minute run and step test for Debre Markos preparatory school 15 sedentary female students.

\begin{tabular}{|l|l|l|l|l|}
\hline $\begin{array}{l}\text { Dependent } \\
\text { variables }\end{array}$ & $\begin{array}{l}\text { Mean value before } \\
\text { training }\end{array}$ & $\begin{array}{l}\text { Mean value during } \\
\text { training }\end{array}$ & $\begin{array}{l}\text { Mean value after } \\
\text { training }\end{array}$ \\
\hline 12 minute run test & 1200.2 & 1708.46 & 2027.06 \\
\hline Step test & 123.06 & 101.33 & 94.93 \\
\hline
\end{tabular}

According to table 5: there was significant difference between pre to post-test score and performance was due to aerobic exercise in which they were engaged in. the mean score value for 12 minute run test before selective aerobic exercise was 1200.2 meter, during training test which was measured at the $4^{\text {th }}$ week of aerobic exercise was 1708.46 meter and after training test which was measured after $8^{\text {th }}$ week of aerobic exercise was 2027.06 meter. When we compared the mean value score before training test with the mean score value of after $8^{\text {th }}$ week aerobic exercise, the mean difference value increased by 826.86 meter $(69 \%)$. This result indicated that, the effective change was observed on participants' cardiovascular fitness level.

The mean score value for step test before training was $123.06 \mathrm{~min} /$ beat, during training test was $101.33 \mathrm{~min} /$ beat and after training test was $94.93 \mathrm{~min} /$ beat. When we compare the mean value score before training test with the mean score value of 8 weeks aerobic exercise, the mean difference value was reduced by $28.13 \mathrm{~min} /$ beat. This was due to $8^{\text {th }}$ consecutive week's aerobic exercise under which the participants were through 
effective change on the participants' heart rate.

\subsection{Standard Deviation}

Table 4: standard deviation of the above result to mean

\begin{tabular}{|l|l|l|l|}
\hline Dependent variables & $\begin{array}{l}\text { Standard deviation } \\
\text { before training }\end{array}$ & $\begin{array}{l}\text { Standard deviation } \\
\text { during training }\end{array}$ & $\begin{array}{l}\text { Standard deviation } \\
\text { after training }\end{array}$ \\
\hline 12 minute run test & 310.14 & 441.49 & 523.83 \\
\hline Step test & 31.78 & 26.17 & 24.52 \\
\hline
\end{tabular}

According to numerical values indicated in table 6,12 minutes run test before training mean of cardiovascular fitness is deviated positively by 310.14 . during training which is measured at $4^{\text {th }}$ week of aerobic exercise the mean of cardiovascular endurance is deviated positively by 441.49 , and after training which was taken after a two week of aerobic exercise the mean of cardiovascular fitness is deviated positively by 523.83.From the above result we infer that selective aerobic exercise has an increasing effect on cardiovascular fitness.

In step test before training which was measured without training mean of cardiovascular fitness is deviated positively by 31.78 . During training which is measured at $4^{\text {th }}$ week of aerobic exercise the mean of cardiovascular fitness is deviated positively by 26.17 and after training which was taken after 8 week of aerobic exercise the mean of cardiovascular fitness is deviated positively by 24.52. This result indicates that the effective change was observed on participants cardiovascular fitness parameters of heart rate.

\section{Discussion}

To achieve the purpose of the study 15 female students from Debre Markos Preparatory school sedentary female students were selected as a subjects and their age was 16-18 years. Selective aerobic exercise was given for 8 consecutive weeks. The variables selected for this study were cardiovascular fitness. Pre, during and post-test were conducted for all 15 subjects on cardio vascular fitness and the score were recorded and then the collected data was analyzed quantitatively by descriptive statics of central tendency.

A sedentary life style is a type of life style with little or no physical activity. A person living a sedentary life style is often sitting or lying down while engaged in an activity like reading, socializing, watching television, playing video game, or using mobile phone or computer for much of the day. A sedentary life style can potentially contribute to ill health and many preventable cause of death.

Charles B. Corbin.et.al.,(2010) state that, active people increase their life expectancy by two years compared to those who are inactive. Sedentary people experience a 20 percent to two-fold increase in early death compared to active people. They all so notes that physical inactivity, in combination with poor eating patterns, ranks with tobacco use among the leading preventable contributors to death. Sedentary life style and lack of physical activity can contribute for risk factors for anxiety, cardiovascular disease, breast cancer, depression, diabetes and high blood pressure

Linda and Philip,(1991) state that, aerobic exercise is a form of exercise that requires a continuous use of oxygen over an extended period of time. This usually means at list 15 to 30 minutes of continuous exercise and aerobic exercise helps to develop cardio vascular endurance.

Lippincott and Wilkins (2013), recommend that, aerobic exercise always be performed at your target heart rate for cardiovascular benefit. The target heart rate is between 60 to 90 percent of the difference between your resting heart rate and your maximum heart rate. Maximum heart rate is 220 minutes your age and an average resting heart rate is about 70. To achieve and maintain cardiovascular endurance people should exercising 3 to 5 days per week. The exercise session should include 15 to 60 minutes of continuous aerobic activity. The number of minutes depends on the intensity of the activity. The American college of Medicine tends to favor aerobic activity of longer duration and less intensity. When this plan is followed, there is more likely to be a total fitness effect and less likely to be any health hazards (Linda and Philip, 1991)

Aerobic exercise are cardio machines, spinning, running, distance swimming, speed walking, jogging aerobic dancing bicycling and rope skipping. According to Heyward, V.H, 2006, aerobic exercise can prevent the chance of developing some cancers, diabetes, depression, cardiovascular disease and osteoporosis

Aerobic exercise has many benefits for sedentary person. Lippincott and Wilkins (2013) state that, strengthening muscles involved in respiration to facilitate the flow of air in and out of lungs and strengthening and enlarging heart muscle to improve its pumping efficacy and reduce resting heart rate; improving circulation efficiency and reducing blood pressure and increase the total number of red blood cells in the body, facilitating transport of oxygen, improve mental health; including reducing stress and lowering the incidences of depression and reducing the risk for diabetes; improving the ability of muscles to use fats during exercise and enhancing the speed at which muscle recover from high intensity exercise and increase storage of energy molecules such as fat and carbohydrates with in the muscles allowing for increase endurance.

Cardiovascular fitness is affected by many physiological parameters including heart rate, stroke volume, cardiac output and maximum oxygen consumption. Understanding the relationship between cardio respiratory and 
endurance training require a review of changes that occur with increased aerobic or an aerobic capacity. An early review by Frank Moran (2010), As aerobic capacity increase general metabolism rises, muscle metabolism is enhanced, hemoglobin raise, buffers in blood stream increase, venous return is improved and stroke volume is improve.

According to Chaarles B.Corbin Ruth and Grey Welk (2000), the result of cardiovascular fitness conditioning has a direct positive effect on muscular endurance and indirect effects on strength and flexibility. To facilitate optimal delivery of oxygen, the working muscles of a person need to train or participate in activities that will build up the energy stores needed for sport

Aerobic exercise can give long term effects on your cardio respiratory system. As Lasa Johnson (2009), has mention on the effect of aerobic exercise can be an effective way to increase the endurance of your cardio respiratory system by maintaining the aerobic phase of exercise for many ways.

\section{Conclusion}

Based on the major finding of the study, effect of selective aerobic exercise on cardiovascular fitness:

- Moderate aerobic exercise has positive effect on improvement of cardiovascular fitness.

- Regular physical exercise depend on training plan is an increasing effect on cardiovascular fitness.

- Selective aerobic exercise (walking, jogging, rope skipping, long distance running, bicycling, aerobic dance) have significant effect bon improvement of health and wellbeing of sedentary people.

- Aerobic exercise has a positive effect on cardio vascular fitness and this positive effects leads to increase stroke volume at rest, lower resting heart rate and lower blood pressure.

- It also has a positive effect on reducing the risk of chronic disease such as hypertension, diabetes, asthma and other upcoming disease.

\section{References}

Astrand.P.O. (1999). Why Exercise and Kinesiology Important for Aerobic Exeercise. USA

Brick.L (1999) Fitness Aerobic, Fitness Spectrum and Human Kinetics. USA

Charansonney OL,Despresjp (2010). Disease prevention and sedentary life style Psycomatic Medicine, 73(2), 134141.

Chaarles B.Corbin Ruth and Grey Welk (2000). Concept of Physical Fitness. Active life style for wellness $\left(10^{\text {th }}\right.$ ed) Mc Graw-Hill Higher Education USA

Frank Moran (2010) Glossary of Fitness and Health Terms. USA

Heyward,V.H, (2006). Advanced Fitness Assessment and Exercise Prescription, (5 ${ }^{\text {th }}$ ed) human kenetics. USA. Linda Meeks (1999) Health a Wellness Approach Merrill published. USA

Linsa Johnson,(2009) Effect of Aerobic Exercise Live Health. University of Alabama Birmingham USA

Lippincott and Wikins (2013) .Handbook American college of Sport Medicine. USA

Reddy.M (2012) Comparison of Circuit Training Methods of Performance Variables. USA

Rosser.M (2001) Body Fitness and Exercise $2^{\text {nd }}$ ed) USA

Shahana.A.S.N (2016) Effect of Aerobic Exercise Program on Health Related Physical Fitness Components of middle Age Women. USA

Thomasas D. Fahey \&Paul M Insel (2001) Fit and Well. (4 ${ }^{\text {th }}$ ed) Core Concepts \& labs in physical fitness and wellness mayfielf publishing company USA

WHO. (2003) Global Strategy on Diet Physical Activity and Health. Accessed on October 20/2017 
Appendix I

Debre Markos University

College of Natural and Computational Science

Department of Sport Science

Experimental scores of 15 subjects in 12 minutes run test in meters

\begin{tabular}{|l|l|l|l|}
\hline Subjects & \multicolumn{1}{|c|}{ Score of PT } & Score of DT & Score of PoT \\
\hline A & 1225 & 1694 & 2100 \\
\hline B & 1148 & 1688 & 2000 \\
\hline C & 1194 & 1720 & 2010 \\
\hline D & 1220 & 1712 & 2200 \\
\hline E & 1208 & 1730 & 2150 \\
\hline F & 1230 & 1728 & 2800 \\
\hline G & 1285 & 1692 & 1900 \\
\hline H & 1282 & 1690 & 1980 \\
\hline I & 1228 & 1735 & 2015 \\
\hline J & 1212 & 1726 & 2018 \\
\hline K & 1206 & 1712 & 1960 \\
\hline L & 1196 & 1688 & 1880 \\
\hline M & 1168 & 1697 & 2020 \\
\hline N & 1175 & 1678 & 2050 \\
\hline O & 1226 & 1737 & 2115 \\
\hline
\end{tabular}

Key:

- $\quad \mathrm{PT} \rightarrow$ Pre training test which was taken before aerobic exercise;

- $\quad$ DT $\rightarrow$ During training test which is measured at 4 th week of aerobic exercise;

- $\quad \mathrm{PoP} \rightarrow$ Post-test which was taken after $8^{\text {th }}$ week of aerobic exercise;

\section{Appendix II}

Debre Markos University

College of Natural and Computational Science

Department of Sport Science

Experimental scores of 15 subjects in step test of 60 second heart rate

\begin{tabular}{|l|l|l|l|}
\hline Subjects & \multicolumn{1}{|c|}{ Score of PT } & \multicolumn{1}{c|}{ Score of DT } & OcT \\
\hline A & 124 & 100 & 90 \\
\hline B & 129 & 111 & 96 \\
\hline C & 125 & 108 & 95 \\
\hline D & 122 & 98 & 95 \\
\hline E & 121 & 97 & 94 \\
\hline F & 120 & 96 & 93 \\
\hline G & 127 & 108 & 100 \\
\hline H & 128 & 110 & 99 \\
\hline I & 119 & 97 & 94 \\
\hline J & 117 & 96 & 92 \\
\hline K & 118 & 95 & 92 \\
\hline L & 131 & 112 & 100 \\
\hline M & 122 & 97 & 96 \\
\hline N & 123 & 99 & 95 \\
\hline O & 96 & 93 \\
\hline Mean score & 123.06 & 101.33 & 94.93 \\
\hline Standard deviation score & 31.78 & 26.17 & 24.52 \\
\hline
\end{tabular}

Key:

- $\quad$ PT $\rightarrow$ Pre training test which was taken before aerobic exercise;

- $\quad$ DT $\rightarrow$ During training test which is measured at $4^{\text {th }}$ week of aerobic exercise;

- $\mathrm{PoP} \rightarrow$ Post-test which was taken after $8^{\text {th }}$ week of aerobic exercise 